Supporting Information for:

\title{
Synthesis and Reactivity of Cationic Palladium Phosphine Carboxylate Complexes
}

\author{
Natesan Thirupathi, Dino Amoroso, Andrew Bell and John D. Protasiewicz*
}

General. All manipulations were carried out using standard Schlenk or drybox techniques, unless stated otherwise. Solvents were purified by standard procedures. $\mathrm{Pd}\left(\mathrm{O}_{2} \mathrm{CCH}_{3}\right)_{2}(\mathrm{Strem}$, Johnson Matthey), $\mathrm{PCy}_{3}$ (Strem, Aldrich), $\left[\mathrm{Li}\left(\mathrm{OEt}_{2}\right)_{2.5}\right]\left[\mathrm{B}\left(\mathrm{C}_{6} \mathrm{~F}_{5}\right)_{4}\right],\left[\mathrm{Me}_{2}(\mathrm{H}) \mathrm{NPh}\right]\left[\mathrm{B}\left(\mathrm{C}_{6} \mathrm{~F}_{5}\right)_{4}\right]$ (Boulder Scientific) and 4-toluenesulfonic acid·monohydrate (Fischer). Elemental analyses were performed by Robertson Microlit Laboratories Inc. (Madison, NJ.) after drying samples in a Fisher Isotemp $282 \mathrm{~A}$ vacuum oven under vacuum at $35{ }^{\circ} \mathrm{C}$ for $24 \mathrm{~h}$. Proton and ${ }^{13} \mathrm{C}$ NMR spectra were recorded on Varian 300 and Varian 600 NMR spectrometers, and the residual solvent protons signal served as reference signals. Phosphorus-31 NMR spectra were recorded on a Varian 300 spectrometer using $85 \% \mathrm{H}_{3} \mathrm{PO}_{4}$ as the external standard. $\mathrm{All}{ }^{31} \mathrm{P}$ and ${ }^{13} \mathrm{C}$ NMR spectra were proton decoupled. Although the existence of trans- $\left[\left(\mathrm{Cy}_{3} \mathrm{P}\right)_{2} \mathrm{Pd}\left(\mathrm{O}_{2} \mathrm{CCH}_{3}\right)_{2}\right]($ ref. 1a-1e) and trans- $\left[\left({ }^{\mathrm{i}} \mathrm{Pr}_{3} \mathrm{P}\right)_{2} \mathrm{Pd}\left(\mathrm{O}_{2} \mathrm{CCH}_{3}\right)_{2}\right]$ (ref. 1a) was indicated, their complete characterization was not reported.

trans- $\left[\left(\mathrm{Cy}_{3} \mathbf{P}\right)_{2} \mathbf{P d}\left(\mathrm{O}_{2} \mathbf{C C H}_{3}\right)_{2}\right]{ }^{1 \text { a-e }}$ In a two-neck round bottom flask equipped with an addition funnel, a reddish brown suspension of $\mathrm{Pd}\left(\mathrm{O}_{2} \mathrm{CCH}_{3}\right)_{2}(5.00 \mathrm{~g}, 22.3 \mathrm{mmol})$ in dichloromethane $(50.0 \mathrm{~mL})$ was set to stir at $-78^{\circ} \mathrm{C}$. The addition funnel was charged with a dichloromethane solution $(30.0 \mathrm{~mL})$ of $\mathrm{PCy}_{3}(13.12 \mathrm{~g}, 46.78 \mathrm{mmol})$, and this solution was then added dropwise to the stirring suspension over the course of 15 minutes, resulting in a gradual change from reddish brown to yellow. After 1 hour of stirring at $-78{ }^{\circ} \mathrm{C}$, the suspension was allowed to warm to room temperature, stirred for an additional two hours, and then diluted with hexanes (20.0 mL). The yellow solid was then collected by filtration (in air), washed with pentane $(5 \times 10 \mathrm{~mL})$ and dried in vacuo. A second crop was isolated by cooling the filtrate to $0{ }^{\circ} \mathrm{C}$ and filtering, washing and drying. Yield: $15.42 \mathrm{~g}(88 \%)$. Anal. Calcd. for $\mathrm{C}_{40} \mathrm{H}_{72} \mathrm{O}_{4} \mathrm{P}_{2} \mathrm{Pd}: \mathrm{C}, 61.17 ; \mathrm{H}, 9.24 \%$. Found: $\mathrm{C}$, 61.44; $\mathrm{H}, 9.58 .{ }^{31} \mathrm{P}\left\{{ }^{1} \mathrm{H}\right\}$ NMR $\left(\mathrm{CD}_{2} \mathrm{Cl}_{2}\right): \delta 21.3 .{ }^{1} \mathrm{H} \mathrm{NMR}\left(\mathrm{CD}_{2} \mathrm{Cl}_{2}\right): \delta 1.15-1.34$ (br m, $\left.18 \mathrm{H}\right)$, 1.64-1.71 (br m, 18H), 1.80-1.82 (br m, 18H), 1.84 (s, 6H), 1.99 (br d, J = 14.4 Hz, $12 \mathrm{H}) .{ }^{13} \mathrm{C}\left\{{ }^{1} \mathrm{H}\right\}$ $\operatorname{NMR}\left(\mathrm{CDCl}_{3}\right): \delta 23.9,26.7,28.2$ (virtual $t,{ }^{2} \mathrm{~J}_{\mathrm{CP}}+{ }^{4} \mathrm{~J}_{\mathrm{CP}}=5.3 \mathrm{~Hz}$ ), 29.6, 33.2 (virtual $\mathrm{t},{ }^{1} \mathrm{~J}_{\mathrm{CP}}+\mathrm{J}_{\mathrm{CP}}=$ $8.8 \mathrm{~Hz}), 175.3$. 
trans-[($\left.\left.{ }^{\mathrm{i}} \mathbf{P r}_{3} \mathbf{P}\right)_{2} \mathbf{P d}\left(\mathrm{O}_{2} \mathbf{C C H}_{3}\right)_{2}\right] .{ }^{1 a}$ In a nitrogen filled flask equipped with an addition funnel, a dichloromethane solution $(20 \mathrm{~mL})$ of $\mathrm{P}^{\mathrm{i}} \mathrm{Pr}_{3}(7.14 \mathrm{~g}, 44.6 \mathrm{mmol})$ was added dropwise to a stirred reddish brown suspension of $\mathrm{Pd}\left(\mathrm{O}_{2} \mathrm{CCH}_{3}\right)_{2}(5.00 \mathrm{~g}, 22.3 \mathrm{mmol})$ in dichloromethane $(30 \mathrm{~mL})$ at $-78^{\circ} \mathrm{C}$. The suspension gradually transformed to a yellow green solution which was allowed to warm to room temperature, stirred for two hours and then filtered through a $0.45 \mu \mathrm{m}$ filter. Concentration of the filtrate to approximately $10 \mathrm{~mL}$ followed by addition of hexanes $(20 \mathrm{~mL})$ afforded yellow solids which were filtered off in air, washed with hexanes $(5 \times 5 \mathrm{~mL})$ and dried in vacuo. Yield: $10.94 \mathrm{~g}(90 \%)$. Anal. Calcd. for $\mathrm{C}_{22} \mathrm{H}_{48} \mathrm{O}_{4} \mathrm{P}_{2} \mathrm{Pd}: \mathrm{C}, 48.49 ; \mathrm{H}, 8.88 \%$. Found: $\mathrm{C}$, 48.55; $\mathrm{H}, 8.85 .{ }^{1} \mathrm{H}$ NMR $\left(\mathrm{CD}_{2} \mathrm{Cl}_{2}\right): \delta 1.37$ (m, 36H), 1.77 (s, 6H), $2.12(\mathrm{br}, 6 \mathrm{H}) .{ }^{31} \mathrm{P}\left\{{ }^{1} \mathrm{H}\right\} \mathrm{NMR}$ $\left(\mathrm{CD}_{2} \mathrm{Cl}_{2}\right): \delta 33.0 .{ }^{13} \mathrm{C}\left\{{ }^{1} \mathrm{H}\right\}$ NMR $\left(\mathrm{CDCl}_{3}\right): \delta 19.6,23.7$ (virtual t,${ }^{1} \mathrm{~J}_{\mathrm{CP}}+{ }^{3} \mathrm{~J}_{\mathrm{CP}}=9.2 \mathrm{~Hz}$ ), 23.8, 175.9.

trans- $\left[\left(\mathrm{Cy}_{3} \mathbf{P}\right)_{2} \mathbf{P d}\left(\mathrm{O}_{2} \mathbf{C C H}_{3}\right)(\mathbf{M e C N})\right]\left[\mathbf{B}\left(\mathbf{C}_{6} \mathbf{F}_{5}\right)_{4}\right](\mathbf{1 a})$. An acetonitrile $(5.0 \mathrm{~mL})$ solution of $\left[\mathrm{Li}\left(\mathrm{OEt}_{2}\right)_{2.5}\right]\left[\mathrm{B}\left(\mathrm{C}_{6} \mathrm{~F}_{5}\right)_{4}\right](864 \mathrm{mg}, 0.992 \mathrm{mmol})$ was slowly added to a dispersion of trans$\left[\left(\mathrm{Cy}_{3} \mathrm{P}\right)_{2} \mathrm{Pd}\left(\mathrm{O}_{2} \mathrm{CCH}_{3}\right)_{2}\right](764 \mathrm{mg}, 0.972 \mathrm{mmol})$ in acetonitrile $(40 \mathrm{~mL})$. The reaction mixture was stirred for $3 \mathrm{~h}$ and then filtered through a $0.45 \mu \mathrm{m}$ filter. The solvent was removed under vacuum to furnish $1.40 \mathrm{~g}$ of $1 \mathrm{a}(99 \%)$. Anal. Calcd. for $\mathrm{C}_{64} \mathrm{H}_{72} \mathrm{NO}_{2} \mathrm{P}_{2} \mathrm{BF}_{20} \mathrm{Pd} \cdot 1 \mathrm{Et}_{2} \mathrm{O}: \mathrm{C}, 53.71 ; \mathrm{H}, 5.44 ; \mathrm{N}$, 0.92\%. Found: C, 53.85; H, 5.18; N, 0.93. ${ }^{31} \mathrm{P}\left\{{ }^{1} \mathrm{H}\right\}$ NMR $\left(\mathrm{CDCl}_{3}\right): \delta 32.7 .{ }^{1} \mathrm{H} \mathrm{NMR}\left(\mathrm{CDCl}_{3}\right): \delta$ 1.12-1.22 (m, 12H), $1.28(\mathrm{qt}, \mathrm{J}=12.9 \mathrm{~Hz}, 3.2 \mathrm{~Hz}, 6 \mathrm{H}), 1.62(\mathrm{q}, \mathrm{J}=12.45 \mathrm{~Hz}, 12 \mathrm{H}), 1.77$ (d, J = $12.6 \mathrm{~Hz}, 6 \mathrm{H}), 1.89$ (d, J = 13.8 Hz, 14H), 1.93 (d, J = $11.4 \mathrm{~Hz}, 16 \mathrm{H}), 2.00$ (s, 3H), 2.39 (s, 3H). ${ }^{13} \mathrm{C}\left\{{ }^{1} \mathrm{H}\right\}$ NMR $\left(\mathrm{CDCl}_{3}\right): \delta 3.30,23.4,26.3,27.9$ (virtual t, ${ }^{2} \mathrm{~J}_{\mathrm{CP}}+{ }^{4} \mathrm{~J}_{\mathrm{CP}}=9 \mathrm{~Hz}$ ), 29.9, 33.7 (virtual t, $\left.{ }^{1} \mathrm{~J}_{\mathrm{CP}}+{ }^{3} \mathrm{~J}_{\mathrm{CP}}=9.45 \mathrm{~Hz}\right), 124.5(\mathrm{br}), 127.2,136.4\left(\mathrm{~d},{ }^{1} \mathrm{~J}_{\mathrm{CF}}=242 \mathrm{~Hz}\right), 138.4\left(\mathrm{~d},{ }^{1} \mathrm{~J}_{\mathrm{CF}}=244 \mathrm{~Hz}\right), 148.4$ $\left(\mathrm{d},{ }^{1} \mathrm{~J}_{\mathrm{CF}}=243 \mathrm{~Hz}\right), 175.5$.

trans- $\left[\left({ }^{\mathrm{i}} \mathbf{P r}_{3} \mathbf{P}\right)_{2} \mathbf{P d}\left(\mathbf{O}_{2} \mathbf{C C H}_{3}\right)(\mathbf{M e C N})\right]\left[\mathbf{B}\left(\mathbf{C}_{6} \mathbf{F}_{5}\right)_{4}\right](\mathbf{1 b})$. An acetonitrile solution $(10 \mathrm{~mL})$ of $\left[\mathrm{Li}\left(\mathrm{OEt}_{2}\right)_{2.5}\right]\left[\mathrm{B}\left(\mathrm{C}_{6} \mathrm{~F}_{5}\right)_{4}\right](960 \mathrm{mg}, 1.102 \mathrm{mmol})$ was slowly added to a stirred acetonitrile solution $(20.0 \mathrm{~mL})$ of trans- $\left[\left({ }^{\mathrm{i}} \mathrm{Pr}_{3} \mathrm{P}\right)_{2} \mathrm{Pd}\left(\mathrm{O}_{2} \mathrm{CCH}_{3}\right)_{2}\right](599 \mathrm{mg}, 1.099 \mathrm{mmol})$. Stirring was continued for additional $4 \mathrm{~h}$, during which time a precipitate formed. The reaction mixture was filtered through a $0.45 \mu \mathrm{m}$ filter and the solvent was removed under vacuum. The remaining material was triturated with pentane $(10.0 \mathrm{~mL})$ and dried under vacuum to give $1.320 \mathrm{~g}$ of $\mathbf{1 b}(99 \%)$. Compound $\mathbf{1 b}$ can also be generated in quantitative yield (as shown by ${ }^{31} \mathrm{P}$ NMR spectroscopy) from the reaction of trans- $\left[\left({ }^{\mathrm{i}} \mathrm{Pr}_{3} \mathrm{P}\right)_{2} \mathrm{Pd}\left(\mathrm{O}_{2} \mathrm{CCH}_{3}\right)_{2}\right]$ and $\left[\mathrm{Me}_{2}(\mathrm{H}) \mathrm{NPh}\right]\left[\mathrm{B}\left(\mathrm{C}_{6} \mathrm{~F}_{5}\right)_{4}\right]$ in acetonitrile $(2$ hr). Anal. Calcd. for $\mathrm{C}_{46} \mathrm{H}_{48} \mathrm{NO}_{2} \mathrm{P}_{2} \mathrm{BF}_{20} \mathrm{Pd}$ : C, 45.81; H, 4.01; N, 1.16\%. Found: C, 46.00; H, 3.92; N, 1.18. ${ }^{31} \mathrm{P}\left\{{ }^{1} \mathrm{H}\right\}$ NMR $\left(\mathrm{CDCl}_{3}\right): \delta 44.5 .{ }^{1} \mathrm{H}$ NMR $\left(\mathrm{CDCl}_{3}\right): \delta 1.37(\mathrm{~m}, 36 \mathrm{H}), 1.92(\mathrm{~s}, 3 \mathrm{H})$, 
$2.22(\mathrm{~m}, 6 \mathrm{H}), 2.34(\mathrm{~s}, 3 \mathrm{H}) \cdot{ }^{13} \mathrm{C}\left\{{ }^{1} \mathrm{H}\right\} \mathrm{NMR}\left(\mathrm{CDCl}_{3}\right): \delta 4.2,19.6,23.2,24.5$ (virtual $\mathrm{t},{ }^{1} \mathrm{~J}_{\mathrm{CP}}+{ }^{3} \mathrm{~J}_{\mathrm{CP}}=$ $10.1 \mathrm{~Hz}), 124.4(\mathrm{br}), 128.5,136.9\left(\mathrm{~d},{ }^{1} \mathrm{~J}_{\mathrm{CF}}=248 \mathrm{~Hz}\right), 138.8\left(\mathrm{~d},{ }^{1} \mathrm{~J}_{\mathrm{CF}}=243 \mathrm{~Hz}\right), 148.7\left(\mathrm{~d},{ }^{1} \mathrm{~J}_{\mathrm{CF}}=240\right.$ $\mathrm{Hz}), 176.0$.

$\left[\left(\mathrm{Cy}_{3} \mathbf{P}\right)_{2} \mathbf{P d}\left(\kappa^{2}-\boldsymbol{O}, \boldsymbol{O}^{\prime}-\mathrm{O}_{2} \mathbf{C C H}_{3}\right)\right]\left[\mathbf{B}\left(\mathrm{C}_{6} \mathbf{F}_{5}\right)_{4}\right]$ (2a). Method 1. A dichloromethane solution (25 $\mathrm{mL})$ of $\left[\mathrm{Me}_{2}(\mathrm{H}) \mathrm{NPh}\right]\left[\mathrm{B}\left(\mathrm{C}_{6} \mathrm{~F}_{5}\right)_{4}\right](1.025 \mathrm{~g}, 1.279 \mathrm{mmol})$ was slowly added to a dichloromethane solution $(50.0 \mathrm{~mL})$ of trans- $\left[\left(\mathrm{Cy}_{3} \mathrm{P}\right)_{2} \mathrm{Pd}\left(\mathrm{O}_{2} \mathrm{CCH}_{3}\right)_{2}\right](1.004 \mathrm{~g}, 1.273 \mathrm{mmol})$ at $-35{ }^{\circ} \mathrm{C}$. The temperature of the reaction mixture was slowly raised to room temperature and stirred for $21 \mathrm{~h}$. During the course of the reaction, the color of the reaction mixture became deep orange. The volatiles from the reaction mixture were then removed under reduced pressure to give a paste, to which was added diethyl ether ( $c a 30 \mathrm{~mL}$ ) to induce the formation of an orange powder. The orange powder was collected by filtration, washed with acetonitrile $(10.0 \mathrm{~mL})$, and dried under reduced pressure to furnish $\mathbf{2 a}$ as an air and moisture stable orange solid. Yield: $1.02 \mathrm{~g} \mathrm{(57 \% ).}$ Method 2. Dichloromethane $(5.0 \mathrm{~mL})$ was syringed into the mixture of trans$\left[\left(\mathrm{Cy}_{3} \mathrm{P}\right)_{2} \mathrm{Pd}\left(\mathrm{O}_{2} \mathrm{CCH}_{3}\right)_{2}\right](333 \mathrm{mg}, 424 \mu \mathrm{mol})$ and 4-toluenesulfonic acid $\cdot$ monohydrate $(85.0 \mathrm{mg}$, $446 \mu \mathrm{mol})$. The resulting mixture was stirred for $22 \mathrm{~h} . \mathrm{A}^{31} \mathrm{P}$ NMR spectrum of the reaction mixture revealed a new peak at $\delta 59.0$. A dichloromethane $(2.0 \mathrm{~mL})$ solution of $\left[\mathrm{Li}\left(\mathrm{OEt}_{2}\right)_{2.5}\right]\left[\mathrm{B}\left(\mathrm{C}_{6} \mathrm{~F}_{5}\right)_{4}\right](400 \mathrm{mg}, 459 \mu \mathrm{mol})$ was then introduced into the above reaction mixture, stirred for $5 \mathrm{~min}$, filtered through a medium porosity frit. The volatiles were removed under vacuum to give a foam that was triturated with hexane $(5.0 \mathrm{~mL})$, and dried under reduced pressure to give a yellow solid $(577 \mathrm{mg})$. This solid was then sonicated in the presence of acetonitrile $(2 \times 3 \mathrm{~mL})$, filtered, and dried under reduced pressure to give $471 \mathrm{mg}$ of $\mathbf{2 a}(79 \%)$. Anal. Calcd. for $\mathrm{C}_{62} \mathrm{H}_{69} \mathrm{O}_{2} \mathrm{P}_{2} \mathrm{BF}_{20} \mathrm{Pd}$ : C, 52.99; H, 4.95\%. Found: C, 53.29; H, 5.05. ${ }^{31} \mathrm{P}\left\{{ }^{1} \mathrm{H}\right\} \mathrm{NMR}$ $\left(\mathrm{CDCl}_{3}\right): \delta 59.3 .{ }^{1} \mathrm{H}$ NMR $\left(\mathrm{CDCl}_{3}\right): \delta 1.24-1.34(\mathrm{~m}, 20 \mathrm{H}), 1.66(\mathrm{q}, \mathrm{J}=11.4 \mathrm{~Hz}, 12 \mathrm{H}), 1.80$ (br, $6 \mathrm{H}), 1.90(\mathrm{br}, 12 \mathrm{H}), 1.96(\mathrm{~d}, \mathrm{~J}=13.8 \mathrm{~Hz}, 12 \mathrm{H}), 2.00(\mathrm{~d}, \mathrm{~J}=12.0 \mathrm{~Hz}, 4 \mathrm{H}), 2.04(\mathrm{~s}, 3 \mathrm{H}) .{ }^{13} \mathrm{C}\left\{{ }^{1} \mathrm{H}\right\}$ $\operatorname{NMR}\left(\mathrm{CDCl}_{3}\right): \delta 25.1,25.7,27.2$ (virtual t, ${ }^{2} \mathrm{~J}_{\mathrm{CP}}+{ }^{4} \mathrm{~J}_{\mathrm{CP}}=5.5 \mathrm{~Hz}$ ), 30.2, $34.7(\mathrm{~m}), 124.2(\mathrm{br}), 136.2$ $\left(\mathrm{d},{ }^{1} \mathrm{~J}_{\mathrm{CF}}=248 \mathrm{~Hz}\right), 138.1\left(\mathrm{~d},{ }^{1} \mathrm{~J}_{\mathrm{CF}}=242 \mathrm{~Hz}\right), 148.2\left(\mathrm{~d},{ }^{1} \mathrm{~J}_{\mathrm{CF}}=239 \mathrm{~Hz}\right), 194.9$.

$\left[\left({ }^{\mathrm{i}} \mathrm{Pr}_{3} \mathbf{P}\right)_{2} \mathbf{P d}\left(\mathrm{\kappa}^{2}-\mathrm{O}, \mathrm{O}^{\prime}-\mathrm{O}_{2} \mathrm{CCH}_{3}\right)\right]\left[\mathrm{B}\left(\mathrm{C}_{6} \mathrm{~F}_{5}\right)_{4}\right](\mathbf{2 b})$. Method 1. A mixture of trans$\left[\left({ }^{\mathrm{i}} \mathrm{Pr}_{3} \mathrm{P}\right)_{2} \mathrm{Pd}\left(\mathrm{O}_{2} \mathrm{CCH}_{3}\right)_{2}\right](51.0 \mathrm{mg}, 93.6 \mu \mathrm{mol})$ and $\left[\mathrm{Me}_{2}(\mathrm{H}) \mathrm{NPh}\right]\left[\mathrm{B}\left(\mathrm{C}_{6} \mathrm{~F}_{5}\right)_{4}\right](76.0 \mathrm{mg}, 94.8 \mu \mathrm{mol})$ in dichloromethane $(5.0 \mathrm{~mL})$ was stirred at room temperature for $24 \mathrm{~h}$. Removal of the volatile materials under reduced pressure yielded an orange gummy material. The ${ }^{31} \mathrm{P}$ NMR spectrum of 
the material revealed the presence of $\mathbf{2} \mathbf{b}(\delta 70)$ and many other unidentified products. Attempts to isolate $\mathbf{2} \mathbf{b}$ from the this reaction mixture were not successful.

Method 2. Dichloromethane $(7.0 \mathrm{~mL})$ was syringed into a mixture of trans-

$\left[\left({ }^{\mathrm{i}} \mathrm{Pr}_{3} \mathrm{P}\right)_{2} \mathrm{Pd}\left(\mathrm{O}_{2} \mathrm{CCH}_{3}\right)_{2}\right](378 \mathrm{mg}, 694 \mu \mathrm{mol})$ and 4-toluenesulfonic acid·monohydrate $(137 \mathrm{mg}, 720$ $\mu \mathrm{mol}$ ), and the mixture was stirred for $22 \mathrm{~h} . \mathrm{A}^{31} \mathrm{P}$ NMR spectrum of the reaction mixture revealed a new peak at $\delta 70$ (major) and smaller peaks at $\delta 37.1$ and 54.0; no signal was observed for trans- $\left[\left({ }^{\mathrm{i}} \mathrm{Pr}_{3} \mathrm{P}\right)_{2} \mathrm{Pd}\left(\mathrm{O}_{2} \mathrm{CCH}_{3}\right)_{2}\right]$. A dichloromethane $(4.0 \mathrm{~mL})$ solution of $\left[\mathrm{Li}\left(\mathrm{OEt}_{2}\right)_{2.5}\right]\left[\mathrm{B}\left(\mathrm{C}_{6} \mathrm{~F}_{5}\right)_{4}\right]$ (628 mg, $720 \mu \mathrm{mol})$ was then introduced into the reaction mixture. After having stirred for 5 minutes, solvent was removed under reduced pressure to give an orange solid. The orange solid was sonicated twice in the presence of diethyl ether $(5 \mathrm{~mL})$ to induce precipitation of a yellow powder. This powder was collected by filtration and dried under vacuum to furnish air and moisture stable 2b. Yield: $645 \mathrm{mg}(80 \%)$. Anal. Calcd. for $\mathrm{C}_{44} \mathrm{H}_{45} \mathrm{O}_{2} \mathrm{P}_{2} \mathrm{PdBF}_{20}: \mathrm{C}, 45.36 ; \mathrm{H}$, 3.89\%. Found: C, 45.37; H, 3.88. ${ }^{31} \mathrm{P}\left\{{ }^{1} \mathrm{H}\right\} \mathrm{NMR}\left(\mathrm{CDCl}_{3}\right): \delta 69.4 .{ }^{1} \mathrm{H} \mathrm{NMR}\left(\mathrm{CDCl}_{3}\right): \delta 1.45(\mathrm{~m}$, 36H), 2.02 (s, 3H), 2.26-2.39 (m, 6H) ${ }^{13} \mathrm{C}\left\{{ }^{1} \mathrm{H}\right\} \mathrm{NMR}\left(\mathrm{CDCl}_{3}\right)$ : $\delta$ 20.1, 25.3, $26.3(\mathrm{~m}), 124.4$ (br), $136.9\left(\mathrm{~d},{ }^{1} \mathrm{~J}_{\mathrm{CF}}=241 \mathrm{~Hz}\right), 138.8\left(\mathrm{~d},{ }^{1} \mathrm{~J}_{\mathrm{CF}}=243 \mathrm{~Hz}\right), 148.8\left(\mathrm{~d},{ }^{1} \mathrm{~J}_{\mathrm{CF}}=237 \mathrm{~Hz}\right), 196.1$.

Reaction of $1 \mathbf{b}$ with acetonitrile- $\boldsymbol{d}_{\mathbf{3}}$. Complex $\mathbf{1 b}(55 \mathrm{mg}, 47 \boldsymbol{\mu m o l})$ was dissolved in acetonitrile$d_{3}(0.79 \mathrm{~mL})$ in air and stored in the same solvent until all $\mathbf{1 b}$ was consumed and cyclometalation was complete as revealed by ${ }^{31}$ P NMR spectroscopy (see below). Subsequently, acetonitrile- $d_{3}$ was removed under vacuum to furnish the oil. NMR $\left({ }^{1} \mathrm{H}\right.$ and $\left.{ }^{31} \mathrm{P}\right)$ spectrum of the above oil reveal the presence of complexes $\mathbf{1 b}$ and $c i s-\left[\left({ }^{\mathrm{i}} \mathrm{Pr}_{3} \mathrm{P}\right) \mathrm{Pd}\left(\kappa^{2}-P, C-\mathrm{P}^{\mathrm{i}} \mathrm{Pr}_{2} \mathrm{CMe}_{2}\right)\left(\mathrm{CD}_{3} \mathrm{CN}\right)\right]\left[\mathrm{B}\left(\mathrm{C}_{6} \mathrm{~F}_{5}\right)_{4}\right]\left(\mathbf{3 a}-\boldsymbol{d}_{\mathbf{3}}\right)$ in approximately 70 and $30 \%$ yield.

Reaction of $1 \mathrm{~b}$ with acetonitrile- $\boldsymbol{d}_{3}$ in presence of sodium carbonate. Sodium carbonate (191 $\mathrm{mg}, 1.806 \mathrm{mmol})$ was added to an acetonitrile- $d_{3}$ solution $(3.5 \mathrm{~mL})$ of $\mathbf{1 b}(162 \mathrm{mg}, 0.139 \mathrm{mmol})$ in air and the resulting heterogeneous mixture was stirred for 15 hours at room temperature. The reaction mixture was filtered and volatiles from the filtrate were removed under vacuum to give a waxy material of $\mathbf{3 a}-\boldsymbol{d}_{\mathbf{3}}$ in $97.4 \%$ yield $(155 \mathrm{mg}, 0.135 \mathrm{mmol})$. Elemental analysis could not be obtained for 3a- $\boldsymbol{d}_{\mathbf{3}}$ because of its waxy nature and difficulty in obtaining the isolable solid. ${ }^{31} \mathrm{P}\left\{{ }^{1} \mathrm{H}\right\}$ NMR $\left(\mathrm{CD}_{3} \mathrm{CN}\right): \delta 51.7$ (d, non-metallated phosphorus), 43.2 (d, metallated phosphorus), ${ }^{2} \mathrm{~J}_{\mathrm{PP}}=30.2 \mathrm{~Hz} \cdot{ }^{31} \mathrm{P}\left\{{ }^{1} \mathrm{H}\right\}$ NMR (THF- $\left.d_{8}\right): \delta 52.4$ (br, non-metallated phosphorus), 44.0 (br, metallated phosphorus). ${ }^{1} \mathrm{H}$ NMR (THF- $\left.d_{8}\right): \delta 1.29\left(\mathrm{~m}, 18 \mathrm{H}, \mathrm{CH}\left(\mathrm{CH}_{3}\right)_{2}\right), 1.46(\mathrm{dd}, \mathrm{J}=17.4 \mathrm{~Hz}$, $15.3 \mathrm{~Hz}, 6 \mathrm{H}, \mathrm{CH}\left(\mathrm{CH}_{3}\right)_{2}$ and d, J = 17.4 Hz, $6 \mathrm{H}$, ring- $\left.\mathrm{C}\left(\mathrm{CH}_{3}\right)_{2}\right), 1.63(\mathrm{dd}, \mathrm{J}=12.7 \mathrm{~Hz}, 9.7 \mathrm{~Hz}, 6 \mathrm{H}$, 
$\left.\mathrm{CH}\left(\mathrm{CH}_{3}\right)_{2}\right), 2.21\left(\mathrm{~m}, 3 \mathrm{H}, \mathrm{CH}\left(\mathrm{CH}_{3}\right)_{2}\right), 2.65\left(\mathrm{~m}, 2 \mathrm{H}, \mathrm{CH}\left(\mathrm{CH}_{3}\right)_{2}\right) .{ }^{13} \mathrm{C}\left\{{ }^{1} \mathrm{H}\right\}$ NMR (THF- $\left.d_{8}\right): \delta 1.33$ (m), 20.1, 20.4 (d, J = 5.1 Hz), 20.5 (m), 21.9, 23.8 (br), 45.7 (br), 125.4 (br), 137.1 (d, ${ }^{1} \mathrm{~J}_{\mathrm{CF}}=$ $242.4 \mathrm{~Hz}), 139.1\left(\mathrm{~d},{ }^{1} \mathrm{~J}_{\mathrm{CF}}=243.0 \mathrm{~Hz}\right), 149.2\left(\mathrm{~d},{ }^{1} \mathrm{~J}_{\mathrm{CF}}=240.6 \mathrm{~Hz}\right)$. No peak was observed for $\mathrm{CD}_{3} \mathrm{CN}$.

Reaction of 3a with acetic acid to generate $\mathbf{1 b}$ To $\mathbf{3 a}-\boldsymbol{d}_{\mathbf{3}}(50 \mathrm{mg}, 43.5 \mu \mathrm{mol})$ in $\mathrm{CDCl}_{3}(0.75$ $\mathrm{mL})$ was added acetic acid $(3 \mu \mathrm{L})$ and $\operatorname{NMR}\left({ }^{1} \mathrm{H}\right.$ and $\left.{ }^{31} \mathrm{P}\right)$ spectra were recorded immediately that indicated formation of $\mathbf{1 b}$ as the only product.

cis-[($\left.\left.{ }^{\mathrm{i}} \mathbf{P r}_{3} \mathbf{P}\right) \mathbf{P d}\left(\boldsymbol{\kappa}^{2}-\boldsymbol{P}, \boldsymbol{C}-\mathbf{P}^{\mathrm{i}} \mathbf{P r}_{2} \mathbf{C M e}_{2}\right)\left(\mathbf{C}_{5} \mathbf{H}_{5} \mathbf{N}\right)\right]\left[\mathbf{B}\left(\mathbf{C}_{6} \mathbf{F}_{5}\right)_{4}\right]$ (3b). Complex $\mathbf{1 b}$ (508 mg, $\left.0.436 \mathrm{mmol}\right)$ was dissolved in dichloromethane $(6.0 \mathrm{~mL})$ and stirred. To the above solution was added dichloromethane $(6.0 \mathrm{~mL})$ solution of pyridine $(164 \mathrm{mg}, 2.073 \mathrm{mmol})$ in air and stirred for 5 hours. The initial light orange color slowly disappeared with the development of a colorless solution. The volatiles from the reaction mixture were removed under vacuum to furnish $\mathbf{1 b}$ in 95\% yield (490 mg, $0.414 \mathrm{mmol}$ ). Crystals were grown by vapor diffusion of heptane into ether solution of $\mathbf{1 b}$ in a NMR tube ( $5 \mathrm{~mm}, 9$ inch) over a period of 3 days. Assignments of the ${ }^{1} \mathrm{H}$ and ${ }^{13} \mathrm{C}$ peaks were unambiguously made with the aid of two dimensional HMQC, HMBC and COSY NMR spectroscopic measurements. ${ }^{31} \mathrm{P}\left\{{ }^{1} \mathrm{H}\right\}$ NMR $\left(\mathrm{CDCl}_{3}\right): \delta 49.1$ (d, non-metallated phosphorus), 37.2 (d, metallated phosphorus), ${ }^{2} \mathrm{~J}_{\mathrm{PP}}=29.3 \mathrm{~Hz} .{ }^{1} \mathrm{H} \mathrm{NMR}\left(\mathrm{CDCl}_{3}\right): \delta 1.14-1.21(\mathrm{~m}$, $24 \mathrm{H}, \mathrm{CH}\left(\mathrm{CH}_{3}\right)_{2}$, ring- $\left.\mathrm{C}\left(\mathrm{CH}_{3}\right)_{2}\right), 1.41-1.47\left(\mathrm{~m}, 12 \mathrm{H}, \mathrm{CH}\left(\mathrm{CH}_{3}\right)_{2}\right), 2.00\left(\mathrm{~m}, 3 \mathrm{H}, \mathrm{CH}\left(\mathrm{CH}_{3}\right)_{2}\right), 2.52(\mathrm{~m}$, $\left.2 \mathrm{H}, \mathrm{CH}\left(\mathrm{CH}_{3}\right)_{2}\right), 7.50\left(\mathrm{t},{ }^{3} \mathrm{~J}_{\mathrm{HH}}=6.3 \mathrm{~Hz}, 2 \mathrm{H}, \mathrm{C}_{5} H_{5} \mathrm{~N}\right), 7.87\left(\mathrm{t},{ }^{3} \mathrm{~J}_{\mathrm{HH}}=7.2 \mathrm{~Hz}, 1 \mathrm{H}, \mathrm{C}_{5} H_{5} \mathrm{~N}\right), 8.51(\mathrm{~d}$, $\left.{ }^{3} \mathrm{~J}_{\mathrm{HH}}=4.2 \mathrm{~Hz}, 2 \mathrm{H}, \mathrm{C}_{5} H_{5} \mathrm{~N}\right) \cdot{ }^{13} \mathrm{C}\left\{{ }^{1} \mathrm{H}\right\} \mathrm{NMR}\left(\mathrm{CDCl}_{3}\right): \delta 20.1,20.3,21.8,22.5,24.6\left(\mathrm{~d},{ }^{1} \mathrm{~J}_{\mathrm{CP}}=13.8\right.$ $\mathrm{Hz}), 24.8\left(\mathrm{~d},{ }^{1} \mathrm{~J}_{\mathrm{CP}}=26.8 \mathrm{~Hz}\right), 40.9\left(\mathrm{dd},{ }^{2} \mathrm{~J}_{\mathrm{PC}}=46.0,28.3 \mathrm{~Hz}, 1 \mathrm{C}\right.$, ring- $\left.C\left(\mathrm{CH}_{3}\right)_{2}\right), 124.1(\mathrm{br}), 126.2$, $136.4\left(\mathrm{~d},{ }^{1} \mathrm{~J}_{\mathrm{CF}}=245.4 \mathrm{~Hz}\right), 138.4\left(\mathrm{~d},{ }^{1} \mathrm{~J}_{\mathrm{CF}}=244.2 \mathrm{~Hz}\right), 138.8,148.4\left(\mathrm{~d},{ }^{1} \mathrm{~J}_{\mathrm{CF}}=237.3 \mathrm{~Hz}\right), 151.1$. Anal. Calcd. for $\mathrm{C}_{47} \mathrm{H}_{46} \mathrm{NP}_{2} \mathrm{PdBF}_{20}$ : C, 47.67; H, 3.92; N, 1.18\%. Found: C, 47.67; H, 3.63; N, 1.17 .

\section{References}

1. (a) Rhodes, L. F.; Vicari, R.; Langsdorf, L. J.; Sobek, A. A.; Boyd, E. P.; Bennett, B. PCT Int. Appl. WO 0350158, 2003. (b) Nomura, K.; Myawaki, T. JP 08104661, 1996. (c) Knifton, J. F. J. Catal. 1979, 60, 27-40. (d) Knifton, J. F. US 4124617, 1978. (e) Neilan, J. P.; Laine, R. M.; Cortese, N; Heck, R. F. J. Org. Chem. 1976, 41, 3455-3460. 